\title{
Analytics for geometric average trigger reset options
}

\author{
Tian-Shyr Dai ${ }^{\mathrm{a}, *}$, Yuh-Yuan Fang ${ }^{\mathrm{b}}$ and Yuh-Dauh Lyuu ${ }^{\mathrm{c}}$ \\ ${ }^{a}$ Department of Applied Mathematics, Chung-Yuan Christian University, \\ 22 Pu-Jen, Pu-chung Li, Chung-Li, Tao-Yuan County, Taiwan 320 \\ ${ }^{\mathrm{b}}$ Analyst, Investment \& Research Division, Fuh-Hua Investment Trust Co., \\ Taipei, Taiwan \\ ${ }^{\mathrm{c}}$ Department of Finance and Department of Computer Science \& \\ Information Engineering, National Taiwan University, No. 1, Taipei, Taiwan
}

The geometric average trigger reset option resets the strike price based on the geometric average of the underlying asset's prices over a monitoring window. Similar contracts have been traded on exchanges in Asia. This paper derives an analytic formula for pricing this option with multiple monitoring windows. The analytic formula in fact is a corollary of a general formula that holds for a large class of path-dependent options: It prices any option whose value can be written as a linear combination of $E\left(\mathrm{e}^{b \cdot X} 1_{\{X \in A\}}\right)$, where $X$ is a multinormal random vector and $b$ is some constant vector. Numerical experiments suggest that the pricing formula approximates the values of arithmetic average trigger reset options accurately. Thus pricing the arithmetic average trigger reset option can benefit from using this formula as the control variate in Monte Carlo simulation. Numerical results also suggest that the geometric average trigger reset option does not have significant delta jump as the standard reset option, and this useful property reduces the hedging risk dramatically.

\section{Introduction}

A reset option is a path-dependent option whose strike price can be reset based on certain criteria. For example, the strike price of a reset call can be reset downward if the underlying asset's price falls below a predetermined value. The underlying asset is assumed to be stock in this paper for convenience. Most reset features embedded in a reset call (put) protect the investors amid declines (increases, respectively) in stock price. This makes a reset option useful to portfolio insurance. To prevent price manipulation, many contracts use the average price of the stock during a certain time period, the so-called monitoring window, as a reset trigger. At the end of each monitoring window lies a reset date. The advantages of using the average price as a reset trigger instead of the stock price alone, as in standard reset options, are (1) to mitigate the possibility of stock price manipulation, especially for thinly traded markets, (2) to provide a strike price correlated with a perceived price trend or fair value, and (3) to lessen the delta jump problem associated with standard reset options. These advantages make the products appealing to some investors. The price that comes with the abovementioned advantages is complexity,

*Corresponding author. E-mail: cameldai@cycu.edu.tw 
Table 1. Geometric and arithmetic average trigger reset call values

\begin{tabular}{lccccc}
\hline Volatility & $50 \%$ & $80 \%$ & $100 \%$ & $120 \%$ & $150 \%$ \\
\hline Geometric & 26.165 & 37.781 & 44.971 & 51.562 & 60.460 \\
Arithmetic & 26.105 & 37.653 & 44.784 & 51.424 & 60.162 \\
Difference & $0.23 \%$ & $0.34 \%$ & $0.42 \%$ & $0.27 \%$ & $0.49 \%$
\end{tabular}

Note: The initial stock price is 100 , the strike price is 95 , the risk-free interest rate is $5 \%$, the time to maturity is 1 year, and the length of the monitoring window which ends at year 0.5 is 0.2 year. 'Geometric' and 'Arithmetic' denote the geometric and arithmetic average trigger reset calls, respectively. The value of arithmetic average trigger reset calls are computed by the Monte Carlo simulation based on 1000000 paths. 'Difference' denotes relative price differences.

as the option combines the features of both Asian and reset options. Similar securities have been issued before. For example, Grand Cathay, a securities firm in Taiwan, issued two average reset options on the Taipei Stock Exchange in 1999.

Standard reset options have been investigated before. Gray and Whaley $(1997,1999)$ analyse the S\&P 500 bear market warrant with a single reset and derive an analytic solution for it. Heynen and Kat (1995) discuss discrete lookback options, which are closely related to reset options. For reset options with the average feature, Chang et al. (2004) give a numerical approach for pricing arithmetic average trigger reset options by extending the approximation algorithm of Hull and White (1993). This approach, however, lacks convergence guarantees as mentioned in Forsyth et al. (2002). Kao and Lyuu (2003) present perhaps the most general algorithm for pricing average-trigger-reset-type options. But their approach applies only to discretely monitored options and is not efficient enough for the present case.

Formally, a geometric average trigger reset option is an option that uses the geometric price average in a monitoring window to set the strike price at the end of that monitoring window, the reset date. Cheng and Zhang (2000) derive an analytic formula for the option with a single monitoring window. However, their formula is erroneous, as will be demonstrated later. This paper will establish the formula for geometric average trigger reset options with an arbitrary number of monitoring windows, generalizing Cheng and Zhang (2000). In fact, the analytic formula is a corollary of a general formula that is of independent interest. This much more general formula prices any option whose value can be written as a linear combination of $\mathrm{E}\left(\mathrm{e}^{b \cdot X} 1_{\{X \in A\}}\right)$, where $X$ is a multinormal random vector and $b$ is some constant vector. Vanilla options, geometric Asian options, rainbow options, and quanto options all fall into this category, with closed-form formulas as multiple integrations. Because evaluating a multidimensional integral is a well-known hard computational problem, the approximation solution of Broadie et al. (1997) remains important for these options.

In real markets, the arithmetic average trigger reset options are more frequently traded than the geometric ones. However, no explicit closed-form solution exists for arithmetic average trigger reset options. This paper suggests that the values of geometric and arithmetic average trigger reset options are very close even when the volatility of the stock price is as high as $150 \%$ in Table 1 . Besides, the arithmetic average trigger reset options can be efficiently priced by a quasi-Monte Carlo method, the control variate method (see Liao and Wang, 2002). This method replaces the evaluation of an unknown value (like the value of an arithmetic average trigger reset option) with the evaluation of the difference between this unknown value and a benchmark (like the value of a geometric average trigger reset option) whose value can be easily obtained. The formula defined in this paper provides a good benchmark for the control variate method.

The paper is organized as follows. The geometric average trigger reset option is formally defined in Section II. The analytic formula for pricing geometric average trigger reset options is derived as a corollary of a general analytic pricing formula in Section III. Numerical results are given in Section IV to support our claims. Section V concludes the paper.

\section{Geometric Average Trigger Reset Options}

An option starts at time 0 and matures at $T$. Let $r$ denote the risk-free interest rate, $S(t)$ denote the stock price at time $t$, and $\sigma$ denote the volatility of stock price $S$. It is assumed that $S(t)$ follows

$$
\mathrm{d} S=\mathrm{r} S \mathrm{~d} t+\sigma S \mathrm{~d} W
$$

in a risk-neutral economy, where $W$ is the standard Wiener process. 
Geometric average trigger reset options are reset options whose strike price can be reset to the geometric average of the stock prices over monitoring windows. Consider a general reset option with $m$ reset dates: $t_{1}, t_{2}, \ldots, t_{m}$, where $0 \leq t_{1}<t_{2}<\cdots<$ $t_{m-1}<t_{m} \leq T$. These $m$ monitoring windows are $\left[t_{1}-\ell_{1}, t_{1}\right],\left[t_{2}-\ell_{2}, t_{2}\right], \ldots,\left[t_{m}-\ell_{m}, t_{m}\right]$, where $\ell_{i}$ denotes the length of the $i$ th monitoring window. The geometric price average of the stock during the $i$ th monitoring window is defined as

$$
\operatorname{avg}\left(t_{i}\right)=\exp \left(\frac{1}{\ell_{i}} \int_{t_{i}-\ell_{i}}^{t_{i}} \ln S(t) \mathrm{d} t\right)
$$

Let $K(t)$ be the strike price prevailing at time $t$. Initially, $K(0)=K$, the original strike price. The reset procedure for the call at reset date $t_{i}$ is

$$
K\left(t_{i}\right)= \begin{cases}K\left(t_{i-1}\right) & \text { if } \operatorname{avg}\left(t_{i}\right) \geq K\left(t_{i-1}\right) \\ \operatorname{avg}\left(t_{i}\right) & \text { if } \operatorname{avg}\left(t_{i}\right)<K\left(t_{i-1}\right)\end{cases}
$$

The payoff of the call at the maturity date is $\max \left(S(T)-K\left(t_{m}\right), 0\right)$. Similarly, the reset procedure for the put at time $t_{i}$ is

$$
K\left(t_{i}\right)= \begin{cases}K\left(t_{i-1}\right) & \text { if } \operatorname{avg}\left(t_{i}\right) \leq K\left(t_{i-1}\right) \\ \operatorname{avg}\left(t_{i}\right) & \text { if } \operatorname{avg}\left(t_{i}\right)>K\left(t_{i-1}\right)\end{cases}
$$

and the payoff of the put at the maturity date is $\max \left(K\left(t_{m}\right)-S(T), 0\right)$. This paper focuses on deriving analytical pricing formula for calls; the extension to puts is straightforward.

\section{The Analytic Approach}

A general analytic pricing formula for pricing a large class of path-dependent options is presented below. The analytic formula for geometric average trigger reset options will then be derived as a corollary.

\section{A general formula}

Let $b$ be an $m$-dimensional vector, $X$ be a nondegenerate $m$-dimensional normal random vector, and $A$ be a subspace of the $m$-dimensional space $\Re^{m}$. All vectors such as $b$ and $X$ will be row vectors throughout the rest of the paper. Superscript '*' denotes the transpose of a vector or matrix. The general pricing formula holds for any derivative whose value can be expressed as a linear combination of $\mathrm{E}\left(\mathrm{e}^{b X^{*}} 1_{\{X \in A\}}\right)$. Many sophisticated derivatives fit this category as mentioned in the introduction.

Assume that the mean vector and the covariance matrix of $X$ are $\mu$ and $\Sigma$, respectively. Because $X$ is not degenerate, $\operatorname{det} \Sigma \neq 0$. The probability density function of $X$ is

$$
f(x)=\frac{1}{\left((2 \pi)^{1 / 2}\right)^{m}(\operatorname{det} \Sigma)^{1 / 2}} \mathrm{e}^{-(x-\mu) \Sigma^{-1}(x-\mu)^{*} / 2}
$$

Therefore,

$$
\begin{aligned}
& \mathrm{E}\left(\mathrm{e}^{b X^{*}} 1_{\{X \in A\}}\right) \\
& \quad=\int_{A} \frac{1}{\left((2 \pi)^{1 / 2}\right)^{m}(\operatorname{det} \Sigma)^{1 / 2}} \mathrm{e}^{b x^{*}-(x-\mu) \Sigma^{-1}(x-\mu)^{*} / 2} \mathrm{~d} x
\end{aligned}
$$

Let $a=b \Sigma$. Then

$$
\begin{aligned}
b x^{*}- & (x-\mu) \Sigma^{-1}(x-\mu)^{*} / 2 \\
= & \mu \Sigma^{-1} a^{*}+\left(a \Sigma^{-1} a^{*} / 2\right) \\
& -\left\{[x-(\mu+a)] \Sigma^{-1}[x-(\mu+a)]^{*} / 2\right\}
\end{aligned}
$$

Equation 1 can now be rewritten as

$$
\begin{aligned}
& \frac{1}{(\sqrt{2 \pi})^{m}(\operatorname{det} \Sigma)^{1 / 2}} \mathrm{e}^{\mu \Sigma^{-1} a^{*}} \mathrm{e}^{a \Sigma^{-1} a^{*} / 2} \\
& \times \int_{A} \mathrm{e}^{-[x-(\mu+a)] \Sigma^{-1}[x-(\mu+a)]^{*} / 2} \mathrm{~d} x \\
& =\frac{\mathrm{e}^{\mu b^{*}+b \Sigma b^{*} / 2}}{(\sqrt{2 \pi})^{m}(\operatorname{det} \Sigma)^{1 / 2}} \int_{A} \mathrm{e}^{-[x-(\mu+b \Sigma)] \Sigma^{-1}[x-(\mu+b \Sigma)]^{*} / 2} \mathrm{~d} x
\end{aligned}
$$

The Black-Scholes formula will be derived from Equation 2 as the first application. Let $X=$ $\ln (S(T) / S(0))$ and $A=\{X \mid X \geq \ln (K / S(0))\}$. The mean and the variance of $X$ are $\left(r-\sigma^{2} / 2\right) T$ and $\sigma^{2} T$, respectively. The value of the vanilla call equals

$$
\begin{aligned}
& \mathrm{e}^{-r T} \mathrm{E}(S(T)-K)^{+} \\
&=\mathrm{e}^{-r T}\left[S(0) \mathrm{E}\left(\mathrm{e}^{1 X^{*}} 1_{\{X \in A\}}\right)-K \mathrm{E}\left(\mathrm{e}^{0 X^{*}} 1_{\{X \in A\}}\right)\right] \\
&= S(0) \mathrm{N}\left(\frac{\ln (S(0) / K)+\left(r+\sigma^{2} / 2\right) T}{\sigma(T)^{1 / 2}}\right) \\
&-\mathrm{e}^{-r T} K \mathrm{~N}\left(\frac{\ln (S(0) / K)+\left(r-\sigma^{2} / 2\right) T}{\sigma(T)^{1 / 2}}\right)
\end{aligned}
$$

where $\mathrm{N}(\cdot)$ denotes the distribution function of the standard normal distribution and Equation 9 is used in the second identity.

Equation 2 reduces the problem of evaluating $\mathrm{E}\left(\mathrm{e}^{b X^{*}} 1_{\{X \in A\}}\right)$ to the integration of a multi-dimensional normal distribution over a region $A$. If the region $A$ is already a rectangular polyhedron or if $X$ is a onedimensional normal random vector, the result can be rewritten similar as the above for the vanilla call option. On the other hand, if $A$ is a polyhedron but not a rectangular one, $X$ can be multiplied by a matrix $C$ to change $A$ into a rectangular polyhedron, $A^{\prime}$. This step transforms the pricing formula into one 
involving distribution functions. More precisely, with $Y^{*}=C X^{*}$, Equation 2 becomes

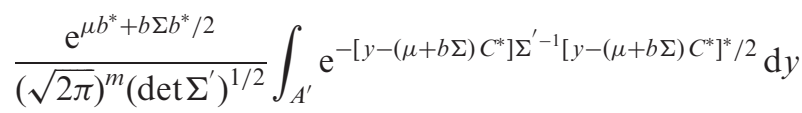

where $\Sigma^{\prime}=C \Sigma C^{*}$.

\section{Analytic formula for geometric average trigger reset options}

Next Equation 3 is used to derive the pricing formula for the geometric average trigger reset call option with $m$ monitoring windows. Define

$$
\begin{aligned}
X_{i} & \equiv \ln \left(\operatorname{avg}\left(t_{i}\right) / S(0)\right), \quad i=1,2, \ldots, m \\
X_{m+1} & \equiv \ln (S(T) / S(0)) \\
X & \equiv\left[X_{1}, X_{2}, X_{3}, \ldots, X_{m+1}\right]
\end{aligned}
$$

$X$ is clearly an $(m+1)$-dimensional normal random vector. The undiscounted value of a reset call is

$$
\begin{aligned}
& \sum_{i=1}^{m}\left[S(0) \mathrm{E}\left(\mathrm{e}^{b_{m+1} X^{*}} 1_{\left\{X \in A_{i}\right\}}\right)-S(0) \mathrm{E}\left(\mathrm{e}^{b_{i} X^{*}} 1_{\left\{X \in A_{i}\right\}}\right)\right] \\
& +S(0) \mathrm{E}\left(\mathrm{e}^{b_{m+1} X^{*}} 1_{\left\{X \in A_{m+1}\right\}}\right)-K \mathrm{E}\left(1_{\left\{X \in A_{m+1}\right\}}\right)
\end{aligned}
$$

where

$$
\begin{aligned}
A_{i}= & \left\{X \mid K\left(t_{m}\right)=\operatorname{avg}\left(t_{i}\right), S(T) \geq \operatorname{avg}\left(t_{i}\right)\right\} \\
& 1 \leq i \leq m \\
A_{m+1}= & \left\{X \mid K\left(t_{m}\right)=K, S(T) \geq K\right\} \\
b_{i}= & {[\overbrace{0, \ldots, 0,}^{i-1} 1, \overbrace{0, \ldots, 0}^{m+1-i}] \quad 1 \leq i \leq m+1 }
\end{aligned}
$$

$X$ 's mean vector $\mu$ is equal to

$$
\begin{aligned}
& {\left[\left(r-\frac{\sigma^{2}}{2}\right)\left(t_{1}-\frac{\ell}{2}\right),\left(r-\frac{\sigma^{2}}{2}\right)\left(t_{2}-\frac{\ell}{2}\right), \ldots,\left(r-\frac{\sigma^{2}}{2}\right)\right.} \\
& \left.\quad \times\left(t_{m}-\frac{\ell}{2}\right),\left(r-\frac{\sigma^{2}}{2}\right) T\right] .
\end{aligned}
$$

Let $\Sigma_{i, j}$ be the covariance of $X_{i}$ and $X_{j}$. Then the elements of the covariance matrix $\Sigma=\left[\Sigma_{i, j}\right]$ are

$$
\begin{aligned}
\Sigma_{i, i}=\sigma^{2}\left(t_{i}-\frac{2 \ell}{3}\right) & \text { if } 1 \leq i \leq m \\
\Sigma_{i, j}=\Sigma_{j, i}=\sigma^{2}\left(t_{i}-\frac{\ell}{2}\right) & \text { if } 1 \leq i<j \leq m+1 \\
\Sigma_{m+1, m+1}=\sigma^{2} T &
\end{aligned}
$$

Consequently, each term in Equation 4 can be reduced to the integration of a multinormal distribution by Equation 2. To state the formula in terms of distribution functions, a matrix is needed for each integration to transform the polyhedral integration area into a rectangular one. This transformation is divided into the two following cases.

Case 1: Area $A_{k}(1 \leq k \leq m)$

Note that each point $X=\left(X_{1}, \ldots, X_{m+1}\right)$ in $A_{k}$ satisfies these $m+1$ inequalities:

$$
\begin{aligned}
X_{k} & \leq \ln (K / S(0)) \\
X_{k}-X_{i} & \leq 0 \quad \text { for } 1 \leq i \leq m+1 \text { and } i \neq k
\end{aligned}
$$

As $A_{k}$ is not rectangular, a matrix $C_{k}$ is needed to linearly transform it into a rectangular one. $C_{k}$ is defined as

$$
\begin{array}{cl}
C_{k}(i, k)=1 & \text { if } 1 \leq i \leq m+1 \\
C_{k}(i, i)=-1 & \text { if } 1 \leq i \leq m+1 \text { and } i \neq k \\
C_{k}(i, j)=0 & \text { otherwise }
\end{array}
$$

where $C_{k}(i, j)$ denotes the element at the $i$ th row and the $j$ th column of $C_{k}$. Define $Y=C X^{*}$. Area $A_{k}$ is hence transformed into

$$
\begin{gathered}
A_{k}^{\prime}=\left\{Y \mid Y_{k} \leq \ln (K / S(0)) \quad Y_{i} \leq 0\right. \text { for } \\
1 \leq i \leq m+1 \text { and } i \neq k\} .
\end{gathered}
$$

Case 2: Area $A_{m+1}$

Note that each point $X=\left(X_{1}, \ldots, X_{m+1}\right)$ in $A_{m+1}$ satisfies these $m+1$ inequalities:

$$
X_{i} \geq \ln (K / S(0)) \quad \text { for } 1 \leq i \leq m+1
$$

Although they define a rectangular polyhedron, a matrix $C_{m+1}$ is required to transform it so the desired formula can be expressed in terms of a distribution function. Let

$$
Y_{i}=-X_{i} \quad \text { for } 1 \leq i \leq m+1
$$

Then the desired $C_{m+1}$ is -1 where $\mathrm{I}$ is the $(m+1) \times(m+1)$ identity matrix. Area $A_{m+1}$ is now transformed into

$A_{m}^{\prime}+1=\left\{Y \mid Y_{i} \leq-\ln (K / S(0)) \quad\right.$ for $\left.1 \leq i \leq m+1\right\}$

After the transformation described above, the undiscounted value of the option now equals

$$
\begin{aligned}
& S(0) \sum_{i=1}^{m}\left\{\mathrm{e}^{r T} \int_{A_{i}^{\prime}} \frac{\mathrm{e}^{-\left[y-\left(\mu+b_{m+1} \Sigma\right) C_{i}^{*}\right] \Sigma_{i}^{-1}\left[y-\left(\mu+b_{m+1} \Sigma\right) C_{i}^{*}\right]^{*} / 2}}{(\sqrt{2} \pi)^{m+1}\left[\operatorname{det} \Sigma_{i}\right]^{1 / 2}} \mathrm{~d} y\right. \\
& -\mathrm{e}^{r\left(t_{i}-\ell / 2\right)-\sigma^{2} \ell / 12} \\
& \left.\quad \times \int_{A_{i}^{\prime}} \frac{\mathrm{e}^{-\left[y-\left(\mu+b_{i} \Sigma\right) C_{i}^{*}\right] \Sigma_{i}^{-1}\left[y-\left(\mu+b_{i} \Sigma\right) C_{i}^{*}\right]^{*} / 2}}{(\sqrt{2} \pi)^{m+1}\left[\operatorname{det} \Sigma_{i}\right]^{1 / 2}} \mathrm{~d} y\right\}
\end{aligned}
$$




$$
\begin{aligned}
& +S(0) \mathrm{e}^{r T} \int_{A_{m+1^{\prime}}} \frac{1}{(\sqrt{2} \pi)^{m+1}\left[\operatorname{det} \Sigma_{m+1}\right]^{1 / 2}} \\
& \times \mathrm{e}^{-\left[y-\left(\mu+b_{m+1} \Sigma\right) C_{m+1}^{*}\right] \Sigma_{m+1}^{-1}\left[y-\left(\mu+b_{m+1} \Sigma\right) C_{m+1}^{*}\right]^{*} / 2} \mathrm{~d} y \\
& -K \int_{A_{m+1^{\prime}}} \frac{\mathrm{e}^{-\left(y-\mu C_{m+1}^{*}\right) \Sigma_{m+1}^{-1}\left(y-\mu C_{m+1}^{*}\right)^{*} / 2}}{(\sqrt{2} \pi)^{m}+1\left[\operatorname{det} \Sigma_{m+1}\right]^{1 / 2}} \mathrm{~d} y
\end{aligned}
$$

where $\Sigma_{i} \equiv C_{i} \Sigma C_{i}^{*}$.

\section{The special case of a single reset}

Next the special case of a single monitoring window is considered. The undiscounted value of this option is Equation 4 with $m=1$. The mean vector of $X$ is

$$
\mu=\left[\left(r-\frac{\sigma^{2}}{2}\right)\left(t_{1}-\frac{\ell}{2}\right),\left(r-\frac{\sigma^{2}}{2}\right) T\right]
$$

The covariance matrix of $X$ is

$$
\Sigma=\left[\begin{array}{cc}
\sigma^{2}\left(t_{1}-\frac{2 \ell}{3}\right) & \sigma^{2}\left(t_{1}-\frac{\ell}{2}\right) \\
\sigma^{2}\left(t_{1}-\frac{\ell}{2}\right) & \sigma^{2} T
\end{array}\right]
$$

The desired transformation matrices $C_{1}$ and $C_{2}$ for integration areas $A_{1}$ and $A_{2}$, respectively, are

$$
C_{1}=\left[\begin{array}{cc}
1 & 0 \\
1 & -1
\end{array}\right], \quad C_{2}=\left[\begin{array}{cc}
-1 & 0 \\
0 & -1
\end{array}\right]
$$

By Equation 3, the integration areas $A_{1}^{\prime}$ and $A_{2}^{\prime}$ are

$$
\begin{aligned}
& A_{1}^{\prime}=\left\{\left(Y_{1}, Y_{2}\right) \mid Y_{1} \leq \ln (K / S(0)), Y_{2} \leq 0\right\} \\
& A_{2}^{\prime}=\left\{\left(Y_{1}, Y_{2}\right) \mid Y_{1} \leq-\ln (K / S(0)), Y_{2} \leq-\ln (K / S(0))\right\}
\end{aligned}
$$

Let $b_{1}=[1,0]$ and $b_{2}=[0,1]$. The undiscounted value of the option is

$$
\begin{aligned}
& S(0) \mathrm{e}^{r T} \mathrm{~N}\left(v_{1}-\left(\mu+b_{2} \Sigma\right) C_{1}^{*}, C_{1} \Sigma C_{1}^{*}\right) \\
& \quad-S(0) \mathrm{e}^{r\left(t_{1}-\ell / 2\right)-\sigma^{2} \ell / 12} \mathrm{~N}\left(v_{1}-\left(\mu+b_{1} \Sigma\right) C_{1}^{*}, C_{1} \Sigma C_{1}^{*}\right) \\
& \quad+S(0) \mathrm{e}^{r T} \mathrm{~N}\left(v_{2}-\left(\mu+b_{2} \Sigma\right) C_{2}^{*}, C_{2} \Sigma C_{2}^{*}\right) \\
& \quad-K \mathrm{~N}\left(v_{2}-\mu C_{2}^{*}, C_{2} \Sigma C_{2}^{*}\right)
\end{aligned}
$$

where $\quad v_{1} \equiv(\ln (K / S(0)), 0), \quad v_{2} \equiv(-\ln (K / S(0))$, $-\ln (K / S(0)))$, and $\mathrm{N}\left(\cdot, \Sigma^{\prime}\right)$ is the cumulative normal distribution function with mean vector 0 and the covariance matrix $\Sigma^{\prime}$.

The floating-strike geometric Asian option has payoffs $\quad\left[S(T)-\exp \left(\int_{0}^{T} \ln S(t) \mathrm{d} t / T\right)\right]^{+}$and $\quad[\exp$ $\left.\left(\int_{0}^{T} \ln S(t) \mathrm{d} t / T\right)-S(T)\right]^{+}$for calls and puts at maturity, respectively (see Angus, 1999). It is a special case of the geometric average trigger reset option with one monitoring window. The claimed correspondence is established by equalizing the reset date with the maturity, the length of the single monitoring window with the life span of the option, and letting the initial strike price to be infinity for the call and zero for the put.

\section{Numerical Results}

Our analytic formula (see Equation 5) is first compared against the formula in Cheng and Zhang (2000). The results are tabulated in Table 2, where 'CZ' denotes the Cheng-Zhang formula and 'Exact' denotes Equation 5. The Monte Carlo simulation ('MC') based on 100 time steps and 1000000 paths as the benchmark. It is apparent that Equation 5 produces values consistent with the Monte Carlo simulation result, whereas Cheng and Zhang's formula does not. This confirms that their formula is incorrect.

The difference between the formula derived and the Cheng-Zhang formula is sketched as follows. The property is used that $\mathrm{E}\left(\mathrm{e}^{b X^{*}} 1_{\{X \in A\}}\right)$ can be reduced to an integration of a multidimensional normal distribution by Equation 1. This significantly reduces the work to derive the analytical formula for geometric average trigger reset options since their values can be viewed as a linear combination of $\mathrm{E}\left(\mathrm{e}^{b X^{*}} 1_{\{X \in A\}}\right)$ (see Equation 4). Although Cheng and Zhang (2000) also show that the value of the option can be written as Equation 4, they derive their formula by complex calculations. Some errors seem to be made in the process. For example, the variance of $(1 / \ell) \int_{t_{1}-\ell}^{t_{1}} W_{u} \mathrm{~d} u$ derived in Lemma B.1 of their paper should be $t_{1}-2 / 3 \ell$ instead of $t_{1}+\ell / 3$, where $W_{u}$ denotes the standard Wiener process.

It is well-known that the delta jump problem makes hedging difficult for standard reset and barrier options. Modifications on standard reset and barrier options are therefore necessary to lessen the problem as surveyed in Hsueh and Liu (2002). The geometric average trigger reset option turns out not to have significant delta jumps. Figure 1 compares the deltas of a vanilla call, a standard reset call ('Standard Reset'), and a geometric average trigger reset call ('Average Reset'). It is evident that the geometric

Table 2. Comparison with the Cheng-Zhang formula

\begin{tabular}{lllr}
\hline Reset date & MC & Exact & CZ \\
\hline 1.00 & 17.189 & 17.254 & 10.866 \\
0.75 & 17.990 & 18.141 & 10.553 \\
0.50 & 18.133 & 18.226 & 9.139 \\
0.25 & 17.936 & 17.847 & 5.567 \\
\hline
\end{tabular}

Note: The initial stock price is 100 , the initial strike price is 95 , the risk-free interest rate is $5 \%$, the volatility is $30 \%$, the time to maturity for the option is one year, and the length of monitoring window is 0.06 year. ' $\mathrm{CZ}$ ' denotes the formula suggests by Cheng and Zhang (2000) and 'Exact' denotes Equation 5. The Monte Carlo simulation ('MC') based on 100 time steps and 1000000 paths is used as the benchmark. The Cheng-Zhang formula fails all four cases. 


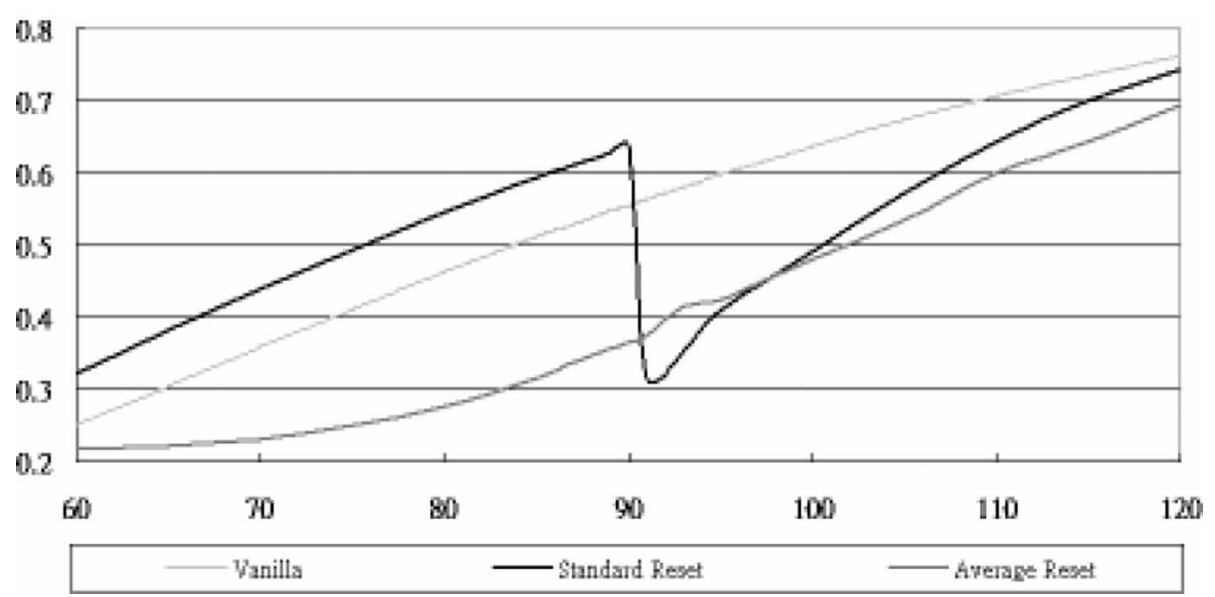

Fig. 1. Deltas of Vanilla Call, Standard Reset Call, and Geometric Average Trigger Reset Call

The options start at year 0 and mature at year 1, the initial stock price is 100 , the strike price is 95 , the risk-free interest rate is $5 \%$, and the volatility is $50 \%$. The standard reset call resets its strike price to 90 once the stock price falls below 90 . The reset date for the geometric average trigger reset call is at year 0.5 . The length of the monitoring window for the geometric average trigger reset call is 0.2 year. The $x$-axis denotes the stock price at year 0 . The $y$-axis denotes the delta. The data for vanilla and standard reset calls are from Hsueh and Liu (2002).

average trigger reset option does not have significant delta jumps as the standard reset option.

\section{Conclusions}

The geometric average trigger reset option resets the strike price based on the geometric average of the stock prices over monitoring windows. Similar contracts have been traded on exchanges in Asia. This paper derives an analytic formula for such options. The formula is in fact a corollary of a much more general formula that is of independent interest as it is applicable to a large class of path-dependent options. Numerical experiments suggest that the pricing formula derived in this paper can estimate the values of arithmetic average trigger reset options very accurately. Pricing the arithmetic average trigger reset options can benefit from using our formula as the control variate in the Monte Carlo simulation. Besides, it is also shown that the geometric average trigger reset option does not have significant delta jumps as the standard reset option. This useful property reduces the hedging risk dramatically.

\section{References}

Angus, J. E. (1999) A note on pricing Asian derivatives with continuous geometric averaging, Journal of Futures Markets, 19, 845-58.
Broadie, M., Glasserman P. and Kou, S. (1997) A continuity correction for discrete barrier options, Mathematical Finance, 7, 325-49.

Chang, C.-C., Chang, S.-L. and Shackleton, M. (2004) Pricing options with American-style average reset features, Quantitative Finance, 4, 292-300.

Cheng, W. Y. and Zhang, S. (2000) The analytics of reset options, Journal of Derivatives, 8, 59-71.

Forsyth, P. A., Vetzal, K. R. and Zvan, R. (2002) Convergence of numerical methods for valuing path-dependent options using interpolation, Review of Derivatives Research, 5, 273-314.

Gray, S. and Whaley, R. (1997) Valuing S\&P 500 bear market warrants with a periodic reset, Journal of Derivatives, 5, 99-106.

Gray, S. and Whaley, R. (1999) Reset put options: valuation, risk characteristics, and an application, Australian Journal of Management, 24, 1-20.

Heynen, R. C. and Kat, H. M. (1995) Lookback options with discrete and partial monitoring of the underlying price, Applied Mathematical Finance, 2, 273-83.

Hsueh, L. P. and Liu, Y. A. (2002) Step-reset options: design and valuation, Journal of Futures Markets, 22, $155-71$.

Hull, J. and White, A. (1993) Efficient procedures for valuing European and American path-dependent options, Journal of Derivatives, 1, 21-31.

Kao, C.-H. and Lyuu, Y.-D. (2003) Pricing of movingaverage-type options with applications, Journal of Futures Markets, 5, 415-40.

Liao, S. L. and Wang, C. W. (2002) Pricing arithmetic average reset options with control variates, Journal of Derivatives, 10, 59-74. 